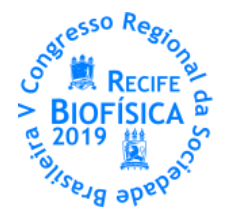

\title{
COMPATIBILIDADE DE PARÂMETROS DO CAMPO DE FORCA GROMOS PARA LIPOPOLISSACARÍDEO COM MODELOS DE AGUA SPC/E, RAIO DE CORTE SIMPLES PARA TERMOS NÃO-LIGADOS E CARGAS ATÔMICAS: PROPRIEDADES DINÂMICAS
}

\author{
Manoela P. M. Lima ${ }^{1 *}$, Manuela Nader ${ }^{1}$, Denys E. S. Santos ${ }^{1}$, Thereza A. Soares ${ }^{1}$ \\ ${ }^{1}$ Grupo de Modelagem de BioMateriais, Departamento de Química Fundamental, UFPE \\ manoelapaschoal@gmail.com;
}

\begin{abstract}
INTRODUÇÃO
0 primeiro conjunto de parâmetros atômicos para lipopolissacarídeos (LPS) foram desenvolvidos e validados [1,2] através de simulações atomísticas de membranas externas bacterianas; $[3,4]$ e de porinas imersa nestas matrizes. [5,6]. Avanços na área de caracterização experimental de estruturas de carboidratos e membranas tornaram necessário o refinamento e reparametrização de um novo conjunto de parâmetros atômicos para membranas LPS compatíveis com os novos resultados experimentais. Com este intuito, um conjunto de parâmetros atômicos específicos para LPS e compatível com o campo de força AMBER foi desenvolvido para reproduzir propriedades estruturais de membranas de LPS do quimiotipo rugoso (Rough LPS) [3]. Este conjunto de parâmetros foi validado através da comparação de simulações da ordem de $1 \mathrm{~s} \mathrm{com}$ medidas experimentais disponíveis na literatura, mostrando um desempenho satisfatório na reprodução de propriedades estruturais, de solvatação e eletrostáticas. Subsequentemente este conjunto de parâmetros foi expandido para a representação de quimiotipos de

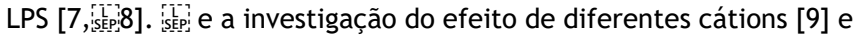
peptídeos antimicrobianos no polimorfismo supramolecular de membranas lipopolissacarídicas [10,11]. Adicionalmente, o campo de força GROMOS foi expandido para a inclusão de parâmetros para membranas lipopolissacarídicas [4,ispi11]. O desenvolvimento e validação dos parâmetros atômicos clássicos através da reprodução criteriosa de medidas experimentais para membranas lipopolissacarídicas possibilitou a investigação de novos fenômenos moleculares. Um exemplo é o efeito de diferentes cátions na estabilidade e dinâmica estrutural de agregados lipopolissacarídicos [9].S[i:?
\end{abstract}

O objetivo do presente trabalho é investigar a compatibilidade de parâmetros do campo de força GROMOS para lipopolissacarídeos com o modelo de água SPC/E usando um raio de corte simples para termos não-ligados e cargas atômicas [12]. Neste pôster serão discutidas propriedades dinâmicas para os sistemas simulados, e enquanto que propriedades estruturais para os mesmos sistemas serão discutidos no pôster apresentado por Manuela Nader e coautores.

\section{MATERIAIS E MÉTODOS}

0 modelo de membrana externa(ME) de bactéria Gram negativa é composta por 72 moléculas lipopolissacarídeas(LPS) pentaaciladas . A parte interna foram adicionadas 180 moléculas de 1-2-dipalmitol3-fosfotidiletanolamina(DPPE), a parte externa foram adicionados $288 \mathrm{Ca}^{2+}$ e os modelos de água. As bicamadas de LPS foram equilibradas até a convergência, por um tempo de 100ns e duplicadas para garantir repetibilidade. Para equilibração do sistema foi utilizado o ensemble NPT com passo de $2 \mathrm{fs}$. Utilizou-se algoritmo LINCS [13] e SETTLE [14] para o comprimento entre as ligações do soluto e a geometria da água. As velocidades iniciais foram extraídas através da distribuição de Maxwell-Boltzmann a $310 \mathrm{~K}$ e 1 bar. 0 tempo de relaxação para o termostato foi de $0,4 \mathrm{ps}$ [15]. A pressão foi mantida a 1 bar nas direções lateral e normal em relação à bicamada por acoplamento fraco a um banho de pressão semi-isotrópico com um tempo de relaxamento de 0,1 ps e compressibilidade isotérmica [16]. As simulações de dinâmica molecular para ME foram simuladas a partir de dois modelos de água distintos, o simple point charge (SPC) [17] e extended simple point charge (SPC/E) [18], a partir dos tratamentos de carga eletrostática: reaction field (RF) [19] usando Unidade Central de Processamento (CPU) e particle Mesh Ewald (PME) [20] com unidade de Processamento gráfico(GPU), resultando em 4 experimentos distintos. As correções de RF para as interações de longa distância foram com raio de corte simples de $1,4 \mathrm{~nm}$ e constante de permissividade de 66 [21-22], enquanto para PME, o raio de corte foi $1,2 \mathrm{~nm}$ com interpolação de $4^{\mathrm{a}}$ ordem de cargas em espaçamento de Fourier de $0,16 \mathrm{~nm}$. A lista de pares não ligados foi atualizada a cada 5 passos para RF e PME. As trajetórias foram gravadas a cada 2 ps. Os solutos contam com a mesma quantidade de átomos. Para manter a mesma dimensão para das caixas de simulação houve alteração na quantidade de átomos para o modelo SPC e SPC/E (i.e. $\mathrm{SPC}=30699$, $\mathrm{SPC} / \mathrm{E}=30507$ ).

Todas as simulações foram realizadas a partir do pacote de programa GROMACS 4.6.7. Os parâmetros atômicos para as interações de van der Waals para carboidratos foram extraídos de GROMOS 45A/53A6 [23] As análises das propriedades foram realizadas através dos programas GROMACS [24] e SUAVE [25].

\section{RESULTADOS E DISCUSSÃO}

Os parâmetros geométricos entre os modelos de água SPC e SPC/E são idênticos, apresentando aumento proporcional nominal nas cargas atômicas de hidrogênio e oxigênio para SPC/E, bem como na energia potencial. Estas características traduzem-se em variações físico-químicas [26], como por exemplo, momento de dipolo e constante de difusão. Pôde-se observar que a quantidade de água SPC dentro da superfície de LPS foi um pouco maior que SPC/E: 876(2,9\%) para SPC-RF, 907 para SPC-PME comparado a 748(2,5\%) para SPC/E-RF e $815(2,7 \%)$ para SPC/E-PME. Para testar a compatibilidade entre os modelos com os parâmetros atômicos GROMOS foram realizadas análises dinâmicas, entre elas a difusividade e o parâmetro de ordem da água. 
A difusividade de íons é uma análise temporal que correlaciona o efeito de transferência de massa das posições em relação ao tempo de simulação. Considerando o sistema previamente equilibrado, ao longo dos intervalos de simulação, não houveram variações significativas na constante de difusão temporalmente para o mesmo modelo de água ou em tratamentos eletrostáticos distintos para o mesmo modelo, como pode ser mostrado na figura 1. Os padrões de difusividade com relação a modelos de água distintos (SPC e SPC/E), mostram maior capacidade difusiva para o modelo SPC em ambos os tratamentos eletrostáticos de longa distância, se comparados com SPC/E. O maior valor de carga atômica nominal entre os átomos de SPC/E faz o sistema mais ordenado e consequentemente menos fluido.

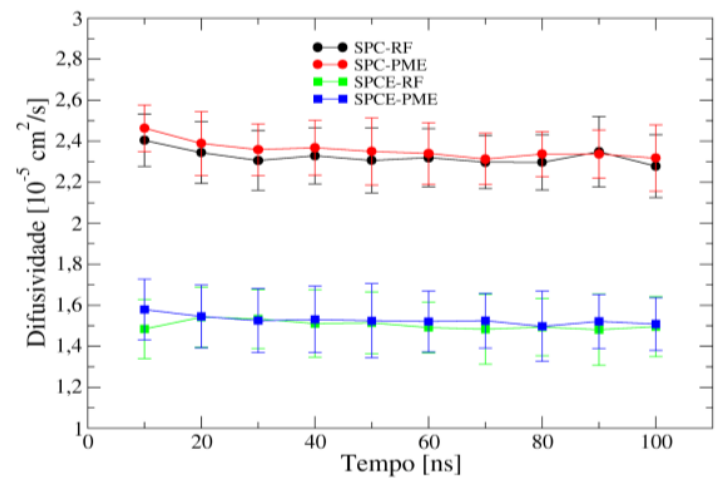

Figura 1.Média dos coeficientes de difusão das moléculas de água em função do tempo. Os sistemas simulados são (SPC-RF) em preto, 2(SPC-PME) em vermelho, 3 (SPC/E-RF) em verde e 4(SPC/E-PME) em azul.

0 parâmetro de ordem trata da orientação média de uma molécula de água em relação ao seu eixo normal. A análise correlaciona o comportamento dos modelos de água em relação aos tratamentos eletrostáticos de longa distância. 0 tratamento eletrostático RF ocorre através de processamento via CPU, enquanto PME é processado em GPU. Em todas as interfaces o comportamento do solvente é randômico de 0 a $2,5 \mathrm{~nm}$ e de 9,5 a $12 \mathrm{~nm}$. A medida que o solvente se aproxima da membrana externa, entre 4,5 a 6,5nm há uma maior ordenação, como mostrado na figura 2. Os maiores picos observados no ponto 5,5 com a presença do LPS. Pode-se perceber aumento do ordenamento aos 10 ns finais da simulação em relação aos iniciais para SPC-RF, SPC-PME E SPC/E-PME. A configuração média inicial e final de SPC-RF e SCP-PME mostram um perfil de $S_{\text {water }}$ similar, também mostrado pelas suas representantes intermediárias (figura 2A -2B).
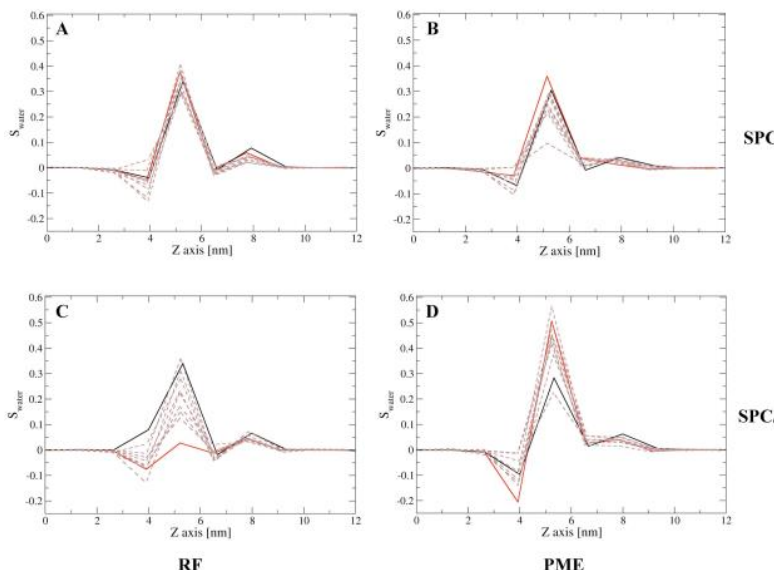

SPC

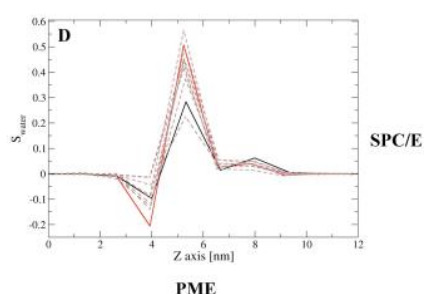

Figura 2. Parâmetro de ordem para as moléculas de água na superfície externa da membrana. As configurações inicial e final são expressas em preto e vermelho, respectivamente. Configurações intermediárias são amostradas a cada 5000 passos e mostradas nas linhas tracejadas em marrom. Os sistemas simulados são A(SPC-RF),B(SPC$\mathrm{PME}), \mathrm{C}(\mathrm{SPC} / \mathrm{E}-\mathrm{RF})$ e $\mathrm{D}(\mathrm{SPC} / \mathrm{E}-\mathrm{PME})$.
Entre SPC/E-RF e SPC/E-PME maiores diferenças podem ser percebidas com respeito ao perfil $S_{\text {water }}$ (figura 2C-2D). S Sater $_{\text {decresce }}$ com o tempo para SPC/E-RF, enquanto cresce para SPC/E-PME. De modo geral, há uma vasta literatura comparando simulações com RF versus PME para sistemas de fase condensada. Constatou-se que PME é melhor parametrizado para tratamento de sistemas periódicos e simulações com íons de alta força iônica, os quais são efetivos na blindagem das interações eletrostáticas e reduz a interação periódica de cópias de soluto. Comparações sistemáticas anteriores de dinâmica molecular mostram que bicamadas de fosfatidilcolina usando extensão do GROMOS 53A6 [27] e o modelo SPC não encontraram diferença significativa entre as propriedades estruturais e dinâmicas derivadas de simulações usando truncamento RF ou PME [28]. De forma geral, os parâmetros atômicos tiveram um desempenho menor quando combinada água SPC/E e correção RF. No entanto, os efeitos da correção PME foram compatíveis aos parâmetros atômicos derivados de GROMOS para LPS na versão GROMACS 2016.

\section{CONCLUSÕES}

A escolha do modelo de água é importante para reprodutibilidade dos sistemas computacionais de forma eficiente. Os dois modelos demonstraram acurácia para descrever as propriedades da água, no entanto o modelo SPC/E mostrou maior permissividade elétrica e coeficiente de difusão próximos aos valores medidos experimentalmente, podendo reproduzir melhor a dinâmica de hidratação das membranas de LPS. SPC apresentou como esperado, uma constante de difusão maior que SPC/E.

Os campos de força são desenvolvidos para reproduzir bem os parâmetros experimentais. O campo de força GROMOS foi parametrizado para a utilização do modelo SPC, do qual SPC/E deriva-se. A última versão GROMACS 2016 não apresenta suporte metodológico para as configurações de validação do GROMOS, no entanto, as simulações realizadas indicam que os parâmetros atômicos derivados de GROMOS para LPS são compatíveis com a versão GROMACS 2016 usando o tratamento de interações de longa distância PME(por processamento GPU ou CPU), utilizando-se um esquema de carga atômica e raio de corte simples para termos nãoligados da função de energia.

\section{REFERÊNCIAS}

LINS, R. D.; STRAATSMA, T. P. Biophys. J. 2001, 81, 1037-1046; SOARES, T. A.; LINS, R. D.; STRAATSMA, T. P. J. Braz. Chem. Soc. 2008, 19, 312-320

KIRSCHNER, K. N.; LINS, R. D.; MAASS, A.; SOARES, T. A. J. Chem. Theory Comput. 2012, 28, 4719-4731

PONTES, F. J. S.; RUSU, V. H.; SOARES, T. A.; LINS, R. D.. J. Chem. Theory Comput. 2012, 8, 3830-3838.

SOARES, T. A.; STRAATSMA, T. P. AIP Conf. Proc. 2007, 963, $1375-$ 1378

STRAATSMA, T. P.; SOARES, T. A. Prot. Struct. Funct. Bionf., 2009, 24, $475-488$

DIAS, R. P. ; DA HORA, G. C.A. ; RAMSTEDT, M. ; SOARES, T. A. J. Chem. Theor. Comp., 2014, 10, 2488-2497;

DIAS, R. P. ; LI, L. ; SOARES, T. A. ; ALEXOV, E. J. Comp. Chem., 2014, 35, 1418-1429

NASCIMENTO, A.; PONTES, F. J.; LINS, R. D.; SOARES, T. A. Chem. Commun. 2014, 50, 231-233

RAVI, H. K.; STACH, M.; SOARES, T. A.; DARBRE, T.; REYMOND, .J. L.; CASCELLA, M. Chem. Commun. 2013, 49, 8821-8823

SANTOS, D. E. S.; POL-FACHIN, L.; LINS, R. D.; SOARES, T. A. J. Chem. Theory Comput. 2017,57,2181-2193

M. P. M. LIMA, M. NADER, D. E. S. SANTOS AND T. A. SOARES J. Braz. Chem. Soc. 2019

MIYAMOTO, S.; KOLLMAN, P. A.; J. Comput. Chem. 1992, 13, 952. 
BUSSI, G.; DONADIO, D.; PARRINelLO, M.; J. Chem. Phys. 2007, 126, 014101.

BERENDSEN, H. J. C.; POSTMA, J. P. M.; DINOLA, A.; HAAK, J. R.; J. Chem. Phys. 1984, 81, 3684.

BERENDSEN, H. J. C.; POSTMA, J. P. M.; VAN GUNSTEREN, W. F.; HERMANS, J. In Intermolecular Forces;Pullman, B.; Reidel: Dordrecht, The Nethelands, 1981.

BERENDSEN, H. J. C.; POSTMA, J. P. M.; DINOLA, A.; HAAK, J. R.; J. Chem. Phys. 1984, 81, 3684.

BerendSen, H. J. C., GRIGeRA, J. R., StRAATSMA, T. P.; J. Phys. Chem. 1987, 91, 6269.

TIRONI, I. G.; SPERB, R.; SMITH, P. E.; VAN GUNSTEREN, W. F.; J. Chem. Phys. 1995, 102, 5451.

DARDEN, T.; YORK, D.; PEDERSEN, L. J. Phys. Chem. 1993, 98, 10089.

ESSEX, J. W.; Mol. Simul. 1998, 20, 159.

GlatTli, A.; DAURA, X.; VAn GUnSteren, W. F. J. Chem. Phys. 2002, 116, 9811.

LINS, R. D.; HUNEnBERGER, P. H. J. Comput. Chem. 2005, 26, 1400. HESS, B.; KUTZNER, C.; VAN DER SPOEL, D.; LINDAHL, E., J. Chem. Theory Comput.2008,4, 435.

SANTOS, D. E. S.; PONTES, F. J. S.; LINS, R. D.; COUTINHO, K.; SOARES, T. A., J. Chem. Inf.Model.2019

BerendSen, H. J. C., GRigera, J. R., StRaAtsma, T. P.; J. Phys. Chem. 1987, 91, 6269.

POGER, D.; VAN GUNSTEREN, W. F.; MARK, A. E. J. Comput. Chem. 2010, 31, 1117.

POGER, D.; MARK, A. E. J. Chem. Theory Comput. 2012, 8, 4807. 